Portland State University

PDXScholar

$11-30-2016$

\title{
Focused-Based Multifractal Analysis of the Wake in a Wind Turbine Array Utilizing Proper Orthogonal Decomposition
}

\author{
Naseem Ali \\ Portland State University \\ Hawwa Falih Kadum \\ Portland State University \\ Raúl Bayoán Cal \\ Portland State University, rcal@pdx.edu
}

Follow this and additional works at: https://pdxscholar.library.pdx.edu/mengin_fac

Part of the Materials Science and Engineering Commons, and the Mechanical Engineering Commons Let us know how access to this document benefits you.

\section{Citation Details}

Ali, N., Falih Kadum, H., \& Bayoán Cal, R. (2016). Focused-based multifractal analysis of the wake in a wind turbine array utilizing proper orthogonal decomposition. Journal Of Renewable \& Sustainable Energy, 8(6), 63306-1-63306-19. doi:10.1063/1.4968032

This Article is brought to you for free and open access. It has been accepted for inclusion in Mechanical and Materials Engineering Faculty Publications and Presentations by an authorized administrator of PDXScholar. Please contact us if we can make this document more accessible: pdxscholar@pdx.edu. 


\section{Focused-based multifractal analysis of the wake in a wind turbine array utilizing proper orthogonal decomposition}

Naseem Ali, Hawwa Falih Kadum, and Raúl Bayoán Cal

Citation: J. Renewable Sustainable Energy 8, 063306 (2016); doi: 10.1063/1.4968032

View online: http://dx.doi.org/10.1063/1.4968032

View Table of Contents: http://aip.scitation.org/toc/rse/8/6

Published by the American Institute of Physics

\section{Articles you may be interested in}

Aerodynamic optimal blade design and performance analysis of $3 \mathrm{MW}$ wind turbine blade with AEP enhancement for low-wind-speed-sites

J. Renewable Sustainable Energy 8, 063303063303 (2016); 10.1063/1.4967971

Publisher's Note: "Aerodynamic optimal blade design and performance analysis of $3 \mathrm{MW}$ wind turbine blade with AEP enhancement for low-wind-speed-sites" [J. Renewable Sustainable Energy 8, 063303 (2016)]

J. Renewable Sustainable Energy 8, 069902069902 (2016); 10.1063/1.4972517

Investigation of the VIMs of a spar-type FOWT using a model test method

J. Renewable Sustainable Energy 8, 063301063301 (2016); 10.1063/1.4966968

A simulation exploration on the grounding characteristic of monopile foundation offshore wind turbines

J. Renewable Sustainable Energy 8, 063304063304 (2016); 10.1063/1.4968242 


\title{
Focused-based multifractal analysis of the wake in a wind turbine array utilizing proper orthogonal decomposition
}

\author{
Naseem Ali, Hawwa Falih Kadum, and Raúl Bayoán Cal \\ Department of Mechanical and Materials Engineering, Portland State University, \\ Portland, Oregon 97207, USA
}

(Received 4 July 2016; accepted 4 November 2016; published online 30 November 2016)

\begin{abstract}
Hot-wire anemometry measurements have been performed in a $3 \times 3$ wind turbine array to study the multifractality of the turbulent kinetic energy dissipation. A multifractal spectrum and Hurst exponents are determined at nine locations downstream of the hub height, bottom and top tips. Higher multifractality is found at $0.5 D$ and $1 D$ downstream of the bottom tip and hub height. The second order of the Hurst exponent and combination factor shows the ability to predict the flow state in terms of its development. Snapshot proper orthogonal decomposition (POD) is used to identify the coherent and incoherent structures and to reconstruct the stochastic velocity signal using a specific number of the POD eigenfunctions. The accumulation of the turbulence kinetic energy in the top tip location exhibits fast convergence compared with the bottom tip and hub height. The dissipation of the large and small scales is determined using the reconstructed stochastic velocities. The higher multifractality is shown in the dissipation of the large scale compared with small scale dissipation showing consistency with the behavior of the original signals. Multifractality of turbulent kinetic energy dissipation in the wind farm is examined and the effect of the reconstructed flow field via proper orthogonal decomposition on the multifractality behavior is investigated. Findings are relevant in wind energy as multifractal parameters identify the variation between the near- and far-wake regions. Published by AIP Publishing.
\end{abstract}

[http://dx.doi.org/10.1063/1.4968032]

\section{INTRODUCTION}

Comprehension of the complex behavior of the wind turbine wake flow is essential to achieve high performance and productivity in wind farms. The dissipation of turbulent kinetic energy and its behavior downstream the turbine is still not fully understood. The major complexity of the wind turbine wake flow lies in the limitation to predict the performance accurately and the lack of ability to identify the activity of the flow scales. ${ }^{1}$ Although the flow within the wind farm has been extensively studied experimentally and computationally, a great portion uses the classical statistical theory to analyze the flow depending on the mean velocity, shear stress, and flux. ${ }^{2-5}$

Mandelbrot introduced an alternative approach, fractal system, to investigate flow fields. ${ }^{6} \mathrm{~A}$ fractal system miniaturizes the whole object or signal to similar fine structures that show geometrically (deterministic) or statistically (random) self-similarity. The flow chaosity can also be identified via fractal structure that characterizes and serves them uniquely as a fingerprint. ${ }^{7}$ Several strategies, which quantify the specifications of the fractal structures, are proposed, such as box counting method, ${ }^{8}$ detrended fluctuation analysis (DFA), ${ }^{9}$ single summation conversion $(\mathrm{SSC})^{10}$ as well as a robust technique known as multifractal wavelet leader (MFWL) developed by Jaffard et al. ${ }^{11}$

Fractal analysis is utilized to investigate different types of turbulent flow some of which are channel flow, ${ }^{12}$ gravity-capillary-waves, ${ }^{13}$ and transport in drift-waves. ${ }^{14}$ In a turbulence field, it is important to examine the intermittent events in fluctuating the turbulence kinetic 
energy dissipation, where intermittency implies a singular behavior and a strong gradient in the flow field signals. Multifractality of the dissipation is proposed by Frisch and Parisi, ${ }^{15}$ where the singularity is quantified through the fractal dimensions. Mandelbrot applied the absolute (Beta) and weighted models to evaluate the multifractality of the energy dissipation. ${ }^{16}$ Meneveau and Sreenivasan ${ }^{17}$ presented a fractal model that fits the entire scaling exponents for the dissipation, and employed the weighted model to construct an artificial signal of the dissipation. Chamorro et al. ${ }^{18}$ used a wavelet framework and structure function to quantify the intermittency and scale-dependent correlation of the wind turbine under neutral stratification. Results showed that the turbine blades amplify the scaling exponents, leading to increase the intermittency. Ali et al. ${ }^{19}$ quantified the intermittency in the velocity signals at the wake of the wind turbine using extend self similarity and compared it with Beta, Kolmogorov, and She-Leveque models. The results showed the scaling exponents are relatively constant in the far-wake regions. Fractal analysis is also used in other disciplines such as the social sciences, geophysics, and medicine. ${ }^{20-25}$ Zunino et al. ${ }^{26}$ used the multifractal spectra to observe the emerging and developed stock markets, where higher multifractality matched the emerging markets. Morales et $a l^{27}$ suggested the stability of the financial firms can be evaluated through multifractal properties.

A fractal system can be categorized as monofractal (homogeneous) or multifractal (heterogeneous), and characterized via the power law with real scaling exponents. Monofractal systems are described by a singular unique scaling exponent, in contrast to the multifractal systems that are labeled by a continuum of scaling exponents. The contribution of the broad distribution function and long-term correlations in large and small fluctuations are responsible for the multifractality in time signals. ${ }^{28}$ Asymptotically, the scaling exponent determines the changes in time intervals and highlights the process mechanism of the flow field. Applying a range of positive and negative moments will provide opportunity to highlight the behavior of large and small fluctuations, respectively. ${ }^{28}$ Scaling exponents showing different behaviors declare that small and large fluctuations are scaled differently. ${ }^{29}$ Therefore, scaling exponents can be utilized in modeling and predicting the future behavior.

Multifractal analysis is applied to quantify the dissipation in the near- and far-wake regions. Proper orthogonal decomposition (POD) is utilized to identify the coherent and incoherent structures. The reconstruction algorithm of the POD is used to rebuild a new time-series data based on the amount of the turbulent kinetic energy. The large scale structures and small scale structures are used separately to reconstruct new flow velocities and thereafter determining the turbulent kinetic energy dissipation of the reconstructed flow field. The contribution of the large and small scales to the energy dissipation is tested after applying the multifractal approach on the reconstructed signal. Thus, the degree of the multifractality of the original and reconstructed signals is compared to show how the POD changes the composition of the signals. The paper is organized as follows: in Sec. II, the theoretical formulation of the multifractal and proper orthogonal decomposition is discussed. In Sec. III, the experimental setup and measurement locations are presented. In Sec. IV, results are described, illustrating the multifractal analysis including spectra, Hurst exponents, combination factor, and thereafter the proper orthogonal decomposition as it pertains to the multifractality analysis of the reconstructed flow field. Finally, conclusions are presented in Sec. V.

\section{THEORY}

\section{A. Multifractal formalism}

The multifractal analysis can be used to detect the fractal properties and scaling behavior of the time series data. Dependent on scales, the power law describes the behavior of a quantity, e.g., velocity, energy dissipation, which can be written as

$$
\frac{\mu_{2}}{\mu_{1}}=\left(\frac{s_{2}}{s_{1}}\right)^{\alpha}
$$


where $\mu_{2}$ and $\mu_{1}$ are statistical measures, $s_{2}$ and $s_{1}$ are scales, and $\alpha$ is the scaling exponent of the power law. For generality, the subscripts are dropped from the scales. Depending on time, $t$, and scales, $s$, the degree of singularity in $\mu$ could be quantified through the Hölder exponents, $h(t)$, as $\mu(t, s) \propto s^{h(t)}$. The distribution of local singularity along the signal can be captured through the singularity spectrum. The MFWL method is used here to find the structure function and thereafter the singularity of the signal. In time-frequency domain, the MFWL method divides the signal into translated and stretched wavelet that should be orthogonal and shows zero mean fast decaying waveform. In this study, the third order Daubechian wavelet, which is a family of the orthogonal wavelets, is used as the dilated and shifted version of the wavelet, ${ }^{30}$

$$
\Psi_{s^{\prime}, i^{\prime}}(t)=\Psi\left(\frac{u-i^{\prime}}{s^{\prime}}\right)
$$

where $\Psi$ is the wavelet, $s^{\prime}$ and $i^{\prime}$ are the dilated and shifted parameter, respectively. The third order Daubechian wavelet has six non-zero scaling coefficients presenting the support function of the wavelet, see Table I.

The convolution operation of the signal $X(t)$ is essentially used to find the wavelet leader, $w(i, s)$, which represents the suprema of the wavelet coefficient, or otherwise expressed as

$$
w(i, s)=\sup _{s^{\prime} \leq s}\left|\frac{1}{N_{s}} \int_{-\infty}^{+\infty} X(t) \cdot \Psi\left(\frac{u-i^{\prime}}{s^{\prime}}\right) d t\right| .
$$

The scaling function based wavelet leader is obtained by

$$
S_{w}(q, s)=\left\{\frac{1}{N_{w}} \sum_{i=1}^{N_{w}}[w(i, s)]^{q}\right\}^{1 / q},
$$

where $N_{w}$ is the number of the wavelet leaders and $q$ is the order. Focused based multifractal analysis introduced by Mukli et $a l^{30}$ is used in this study to estimate the best exact fit that is deduced via convergence of the least sum of squared errors, SSE,

$$
S S E=\sum_{q} \sum_{s}\left[\log S_{w}(q, s)-H(q) \cdot(\log s-\log L)-\log S_{w}(L)\right]^{2},
$$

where $\log$ and $L$ are the logarithm and largest scale, respectively. The scaling behavior can be observed through determining the $q$ order Hurst exponent, $H(q)$, that can be obtained via finding the slope of the regression lines and presents the parameterization of the multifractal structure of the time series data, $S_{w}(q, s) \propto s^{H(q)}$. The Hölder exponents, $h$, are associated with the multifractal Hurst exponents, $H$, and scaling exponents, $\tau$, as follows:

$$
h=\frac{d \tau(q)}{d q}=\frac{d(q \cdot H(q)-1)}{d q} .
$$

The Hurst exponents are used to distinguish the temporal features of the time series data and identified the degree of multifractality, where a monofractal system shows a constant Hurst exponent, by contrast the multifractal signals showing a remarkable dependence of the Hurst exponent on the order, $q$. Di Matteo et al. ${ }^{31}$ investigated the multifractality of a wide range of

TABLE I. Daubechian wavelet coefficients.

\begin{tabular}{lcccccc}
\hline \hline Coefficients & $a_{0}$ & $a_{1}$ & $a_{2}$ & $a_{3}$ & $a_{4}$ & $a_{5}$ \\
\hline Value & 0.33267055 & 0.80689150 & 0.45987750 & -0.1350110 & -0.08544127 & 0.03522629 \\
\hline \hline
\end{tabular}


developing and developed markets, and revealed that the second order of the Hurst exponent can predict the development level of a market. Morales $e t ~ a l . ~{ }^{27}$ utilized the multifractal characterizations as an indicator of financial crises and company stability, and found that the second order of the Hurst exponent, $H(q=2)$, increases when the financial crises begins. The degree of multifractality can be also captured through multifractal spectrum, where the width of the multifractal spectrum is also used as an indictor to the multiscaling process. ${ }^{32-34}$ The singularity spectrum can be obtained from the Legendre-transformation to the singularity or Hölder exponents,

$$
F(h)=\inf _{q}(q h-\tau(q))
$$

Ideally, the singularity spectrum is bounded by two limits at $q= \pm \infty$ and shows a concave function with a parabolic shape. The width and the shape of the spectrum curve contain characteristic information of the tested data set. The parameter $P_{c}=h_{\max } f_{w h m} / F_{\max }$ is a combination of the Hölder exponent at the maximum multifractal spectrum and the full width at half maximum of the spectrum, see Figure 1. $P_{c}$ is used to distinguish the activity of the time series data. ${ }^{35}$

\section{B. Proper orthogonal decomposition}

To objectively quantify the dominant flow scales, proper orthogonal decomposition tool is used to decompose the flow into different scales depending on the turbulent kinetic energy. Lumley ${ }^{36}$ presented a classical POD technique in the turbulent velocity flow field. Thereafter, Sirovich $^{37}$ introduced the snapshot POD to remedy the difficulties of the classical method and save the computational time by reducing the eigenvalue problem to the number of the snapshot instead of the physical mesh. The POD approach is used to investigate different flow disciplines such as wind turbine wake flow, ${ }^{38-40}$ channel flow, ${ }^{41}$ and turbulent boundary layer. ${ }^{42}$ Ukeiley et $a l^{43}$ used POD with the multifractal measures to study the flow in the lobed mixer. They observed that the reconstructed velocity is highly intermittent although the original velocity exhibits low intermittency.

Proper orthogonal decomposition is a mathematical tool that depends on the two-point correlation and identifies the flow scales as a function of the turbulent kinetic energy content. The decomposition is achieved by detecting a deterministic field presenting the maximum projection onto the stochastic velocity. The optimal projection between the stochastic velocity and the deterministic fields, $\phi(t)$, can be performed by finding the maximum inner products. This study follows the POD procedures presented in Ref. 44. The time-series data are partitioned into adjacent portions and the number of snapshots, $N$, is controlled by the total length of the time series

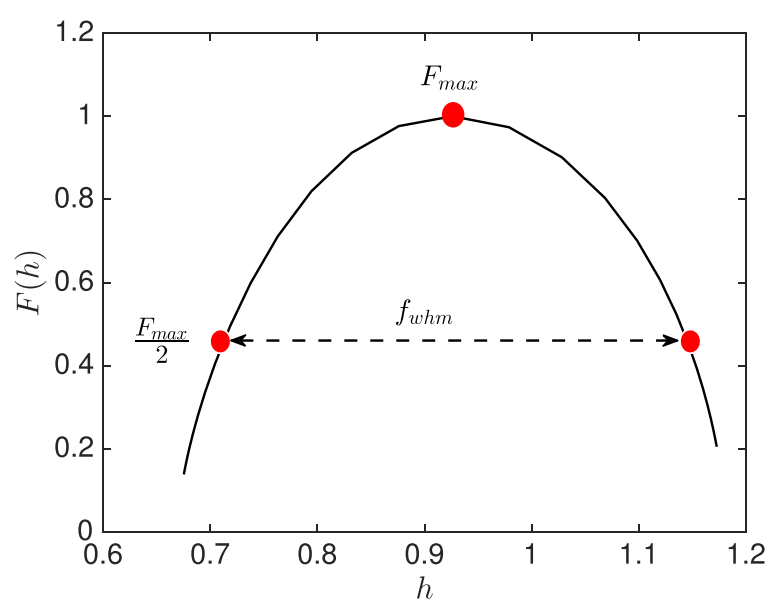

FIG. 1. Theoretical multifractal spectrum. 
data and the time length, $N_{\text {period }}$, at each snapshot. The $N_{\text {period }}$ and $N$ are chosen according to the flow convergence and decorrelation criterions. The kernel of the POD is the covariance matrix and can be determined as

$$
R\left(t, t^{\prime}\right)=\frac{1}{N} \sum_{n=1}^{N} M\left(t^{n}\right) M^{T}\left(t^{\prime n}\right),
$$

where $R\left(t, t^{\prime}\right)$ is a temporal correlation between two times $t$ and $t^{\prime}, M$ is the velocity matrix, and $T$ is the transpose. The zero-mean fluctuating velocities are used to obtain the kernel of the POD. Following the Fredholm integral equation, the maximization of the projection can be achieved in a finite domain, $\Omega$, as

$$
\int_{\Omega} R\left(t, t^{\prime}\right) \phi\left(t^{\prime}\right) d t^{\prime}=\lambda \phi(t)
$$

where $\lambda$ is the eigenvalue. The eigenvalue problem is used to solve the deterministic function that takes the following form:

$$
[C][B]=\lambda[B],
$$

where $[C]$ and $[B]$ are a relative to the correlation matrix and the basis of eigenfunctions, respectively. The diagonal matrix, $\lambda$, is real and non-negative, and corresponds to distinct eigenfunctions. Both eigenvalues and eigenfunctions are arranged in optimal sense. POD modes are determined by projecting the velocity matrix into the eigenvector space and thereafter normalized with $\mathrm{L}^{2}$-norm to acquire the orthonormality basis, ${ }^{38}$

$$
\phi^{n}=\frac{\sum_{n=1}^{N} B^{n} M}{\left\|\sum_{n=1}^{N} B^{n} M\right\|} .
$$

The trace of the eigenvalue matrix indicates the average of the turbulent kinetic energy and can be presented in normalized, $A_{n}$, and cumulative, $B_{n}$, as

$$
\begin{array}{r}
A_{n}=\frac{\lambda_{n}}{\sum_{n=1}^{N} \lambda_{n}}, \\
B_{n}=\frac{\sum_{n=1}^{n} \lambda_{n}}{\sum_{n=1}^{N} \lambda_{n}} .
\end{array}
$$

POD has the ability to rebuild the stochastic velocity, where the number of modes used in the reconstruction operation determines the contained energy of the reconstructed velocity. Using the POD eigenfunctions, the reconstructed velocity can be built as follows:

$$
\vec{u}\left(t^{n}\right)=\sum_{n=1}^{N}\left\{\int_{\Omega} M\left(t^{n}\right) \phi^{n}\left(t^{\prime}\right) d t^{\prime}\right\} \phi^{n}(t),
$$

where $\left\{\int_{\Omega} M\left(t^{n}\right) \phi^{n}\left(t^{\prime}\right) d t^{\prime}\right\}$ is the projection of the velocity field onto POD modes, and presents the principle coefficients that convey the spectral contribution of the modes. 


\section{EXPERIMENTAL SETUP}

The experimental tests on an array of horizontal axis wind turbines were performed at the closed-loop Corrsin wind tunnel at Johns Hopkins University. The dimensions of the wind tunnel test section are $10 \mathrm{~m}, 0.9 \mathrm{~m}$, and $1.2 \mathrm{~m}$ in the streamwise, wall-normal, and spanwise directions, respectively. The entrance of the tunnel is provided by an active grid to generate turbulence. The grid was made of $19 \mathrm{~mm}$ diameter aluminum shafts and distributed vertically and horizontally to seven and five shafts, respectively. The shafts are rotated randomly and individually via $1 / 4 \mathrm{hp}$ AC motors. Winglets of $3.18 \mathrm{~mm}$ thick aluminum plates are attached to the rods and arranged as 8 horizontally and 6 vertically. The inlet mean velocity shear profile was generated using nine strakes of a thick acrylic plate. The strakes were uniformly distributed in the spanwise direction of the wind tunnel. An atmospheric-like boundary layer was furnished by adding a 24-grit aluminum oxide sand paper to the ground. Figure 2 presents the schematic of the experimental setup.

A three-bladed rotor of $12 \mathrm{~cm}$ diameter, $D$, was used as a model of the wind turbine. The rotors were manufactured from a steel sheet of $0.48 \mathrm{~mm}$ thick, and twisted $15^{\circ}$ and $10^{\circ}$ at the root and the tip, respectively. Thus, the rotor was attached to $1 \mathrm{~cm}$ diameter and $10.7 \mathrm{~cm}$ tall tower. The scale of the current turbine model to the real turbines was about 1:830. Nine wind turbines models are distributed as $3 \times 3$ array and placed at $1.5 \mathrm{D}$ from the sidewalls of the tunnel and $3 D$ downstream of the leading edge. The wind turbine array was spaced $7 D$ in the stream-wise and $3 D$ in the spanwise directions.

To include the effect of the wake accumulation through this analysis, the measurements that are considered here only behind the center of the third row of the wind farm. The measurements behind the first two rows are disregarded, although the measurements are different. Time series data of $100 \mathrm{~s}$ length were gathered via X-type hot-wire anemometry at 21 vertical and 9 streamwise locations. Downstream locations beginning at $0.5 D$, and with $1 \mathrm{D}$ spacing from $1 D$ through $8 D$, and the vertical locations starting at $0.5 \mathrm{~cm}$ above the ground moved vertically with an increment of $1 \mathrm{~cm}$. The hot wire was assembled from two platinum-coated tungsten wires of $2.5 \mu \mathrm{m}$ diameter. Time series data were sampled at the frequency of $40 \mathrm{kHz}$ and refined to $10 \mathrm{kHz}$ using a low-pass filter.

Throughout the experimental tests, the free stream velocity was maintained at a constant and the homogeneity of the velocity profile in the spanwise direction showed a reasonable deviation of $0.36 \mathrm{~ms}^{-1}$ in mean velocity and $1 \%$ in normalized Reynolds stress. It is also noted that a boundary layer develops in the top wall of the test section, but it is considered negligible as seen by the wind turbine array. Supplementary details regarding the experimental data, measurement devices, and all statistical analysis can be found in Refs. 2, 19, and 45. This study focuses on three wall-normal locations named bottom tip, hub height, and top tip at nine downstream locations starting from the $0.5 D$ through $8 D$ as shown in Figure 3.

To determine the turbulent kinetic energy dissipation, Meneveau and Sreenivasan ${ }^{46}$ showed that the square of gradient in only one direction can represent the actual rate of the energy dissipation. Chamorro et al. ${ }^{47}$ used the second order of the velocity structure function to determine the turbulent kinetic energy dissipation in the wind farm. They highlighted that the full velocity gradient is required to acquire an accurate estimate of energy dissipation. Meneveau and Sreenivasan $^{46}$ and Chamorro et $a l .{ }^{47}$ used the Taylor frozen field hypothesis to transform the

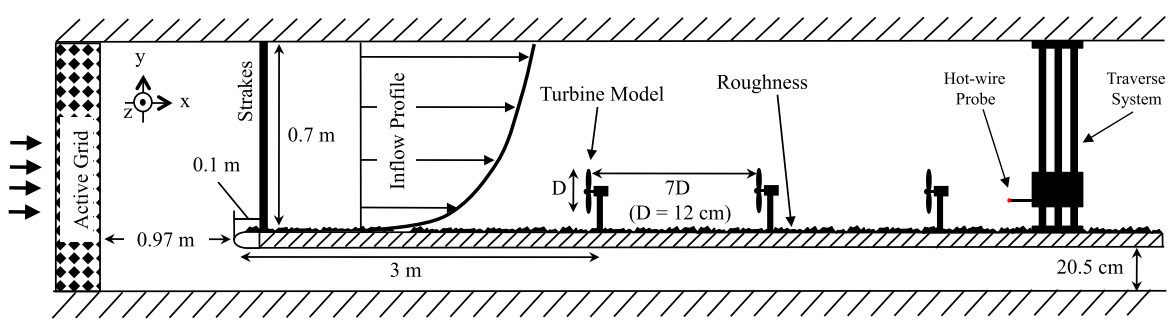

FIG. 2. Side view of the experiment setup. 


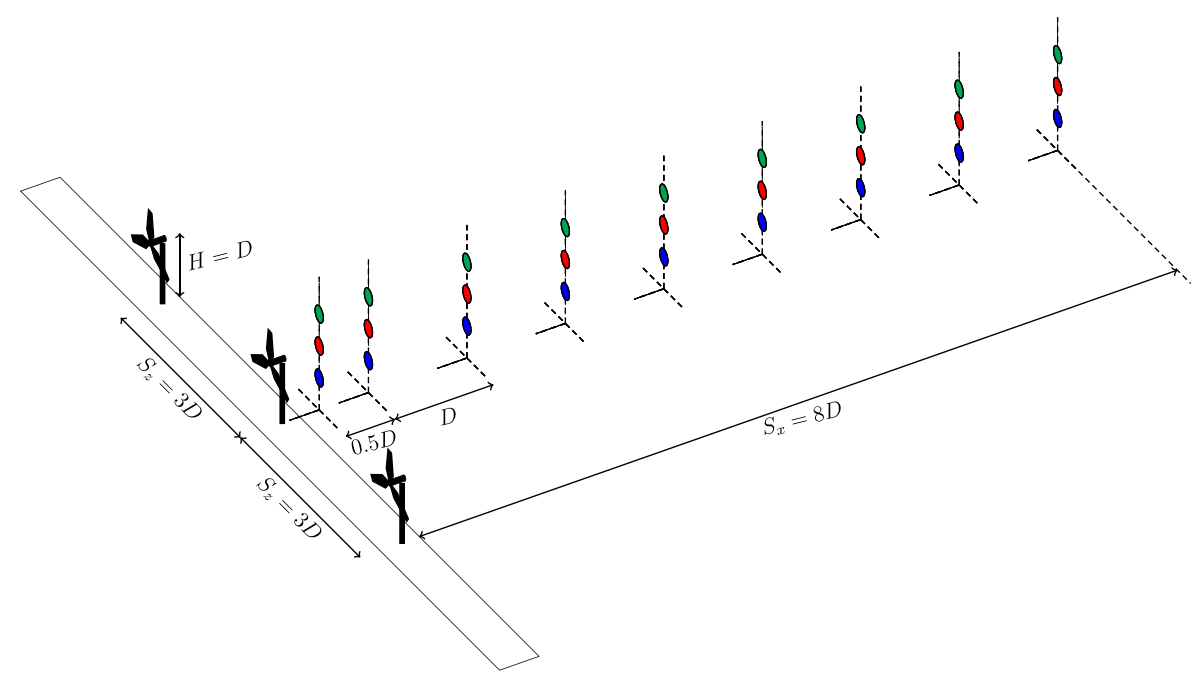

FIG. 3. Schematic of streamwise and wall-normal measurement locations behind the center turbine of the last row in model wind farm array. Bottom tip (blue circles), hub height (red circles), and top tip (green circles).

spatial dependence into temporal. In this study, the velocity gradient approach is used to determine the dissipation as

$$
\epsilon \sim\left(\frac{d u}{d t}\right)^{2}
$$

where $\epsilon$ is the total dissipation, $u$ is the streamwise fluctuating velocity, and $t$ is the time. The first order of central finite difference is used to achieve the gradients.

\section{RESULTS}

\section{A. Multifractal analysis}

Figure 4 contains the multifractal spectra of the turbulent kinetic energy dissipations at bottom tip, hub height, and top tip. The $x, y$, and $z$ axes represent the normalized streamwise direction, $x / D$, singularity spectrum, $F(h)$, and Hölder exponent, $h$, respectively. The singular spectrum differs greatly depending on the physical locations. Bottom tip shows higher Hölder exponents at $0.5 D$, where the spectrum lies between $0.7 \leq h \leq 2$. The spectrum transforms toward moderate Hölder exponents at $1 D$ and continues moving asymptotically toward the small exponents. After $5 D$, the trend remains stable for the following downstream locations. The left tail of the spectrum shifts up with the increasing of $x / D$. In contrast, the right tail

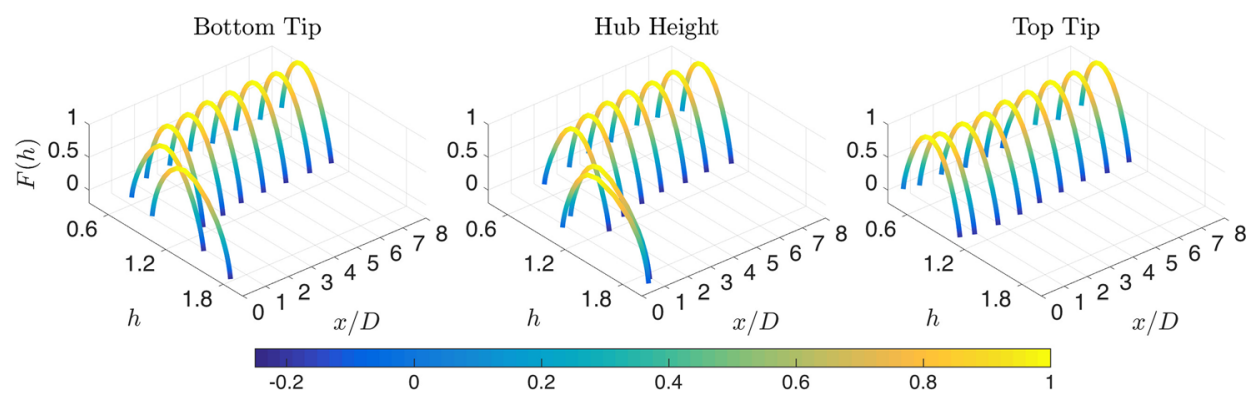

FIG. 4. Distribution of the singularity spectrum, $F(h)$, downstream the bottom tip, hub height, and top tip. The $x / D$ is the streamwise direction and $h$ is the Hölder exponent. The colorbar presents the value of $F(h)$. The near-wake regions and farwake regions can be identified as $(x / D<3)$ and $x / D>5$, respectively. 
moves down at locations downstream of the turbine. The long right tail reflects the sensitivity to the small local fluctuations. ${ }^{9,48}$ At hub height, the multifractal spectrum also moves toward the higher Hölder exponents at $x / D=0.5$ and 1 . Thereafter, the spectrum shifts asymptotically to lower singularity exponents. The left and right tails of the spectrum show the same trend that is noticed at the bottom tip. At the top tip, the multifractal spectrum locates at the same limit of the Hölder exponents with a slight deviation at $x / D=2$ and 3. The left tail of the spectrum shows approximately the same level at $x / D=0.5-4$. Thereafter, the left tail moves up slightly from the previous location level. The minimum Hölder exponent can be used to predict the complexity of the flow, as highlighted by the Seo and Lyu ${ }^{49}$ who concluded that the minimum Hölder exponent decreases with a less complex turbulent flow. Following the thought, here the minimum Hölder exponent decreases with increasing $x / D$, especially at the bottom tip and hub height locations. Maximum Hölder exponents are identified at $0.5 D$ and $1 D$ downstream of the hub height and bottom tip, where the interaction between the wind turbine and the boundary layer is more severe. Macek ${ }^{50}$ pointed out that the shape of the singularity spectrum can be related to the heterogeneity of the energy transfer through scales and the multiscaling nature of the energy cascade. In other words, the shape of the spectrum can be used to reveal if the dissipation is independent of the scales as shown by Kolmogorov ${ }^{51}$ or it is intermittent. Strong multifractality means high intermittency and the dissipation is fully dependent on the scales. Here, the near-weak region displays high intermittency events, which is contrary to observations in the far-wake. Multifractality of the downstream locations might be generated from the multifractal structures of the near-wake regions that are convected downstream. Thus, reducing the multifractality of the downstream locations is a result of increasing the Reynolds number and consequently separation of scales.

Figure 5 presents the Hurst exponent, $H$, for the same locations shown in the previous figure. The order, $q$, is chosen to be between $q= \pm 15$ with increments of unity. The limits of the order, $q$, present the $\pm \infty$ ends of the singularity spectrum..$^{30}$ The multifractality is identified through order dependent Hurst exponents. At the horizontal line of the bottom tip, higher Hurst exponents are encountered at $x / D=0.5$ and 1 , and thereafter for greater $x / D$, the variations with the order are reduced. The same trend is noticed at the hub height locations, where the maximum variations with $q$ occur at downstream locations close to the turbine. The Hurst exponent distribution at $x / D=1$ of hub height is higher than the distribution at bottom tip, affirming the wake effect at hub height as strong and extending a long distance downstream of the rotor. Top tip locations show different behavior, where all the downstream locations show approximately the same distribution with the order.

Figure 6 presents the second order of the Hurst exponent, $H(q=2)$, and the combination parameter, $P_{c}$, for the same considered locations. The second order of Hurst exponents are tested here to characterize and demonstrate changes between the near- and far-wake regions. Furthermore, assessing the development and recovery of the flow is also possible. At bottom tip and hub height, the $H(q=2)$ shows higher values at $x / D=0.5$ and decreases with increasing streamwise direction. The second order of the Hurst exponent at $x / D=0.5$ is approximately two times higher than the exponent at $8 D$. The maximum difference in the spatial distribution of the

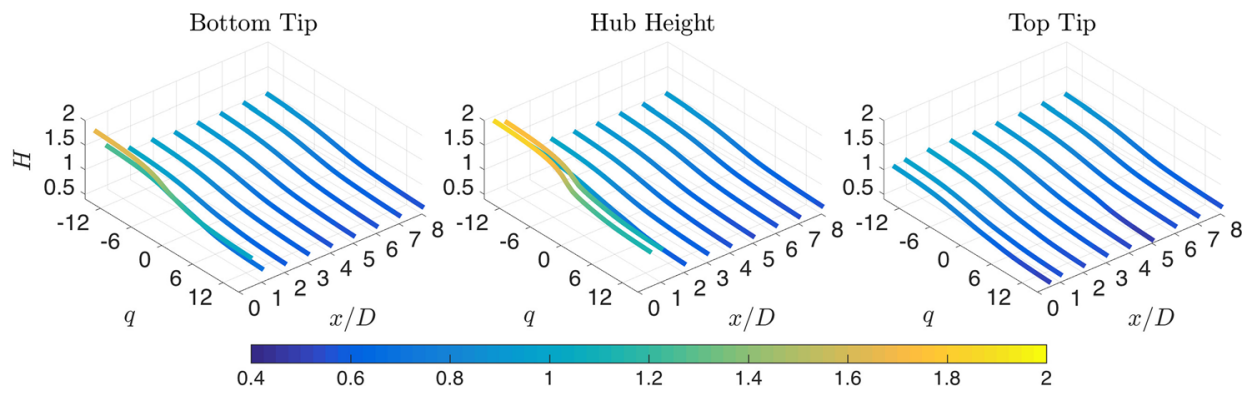

FIG. 5. Distribution of the Hurst exponent, $H$, downstream the bottom tip, hub height, and top tip. The $q$ is the order moment. The colorbar presents the value of $H$. 

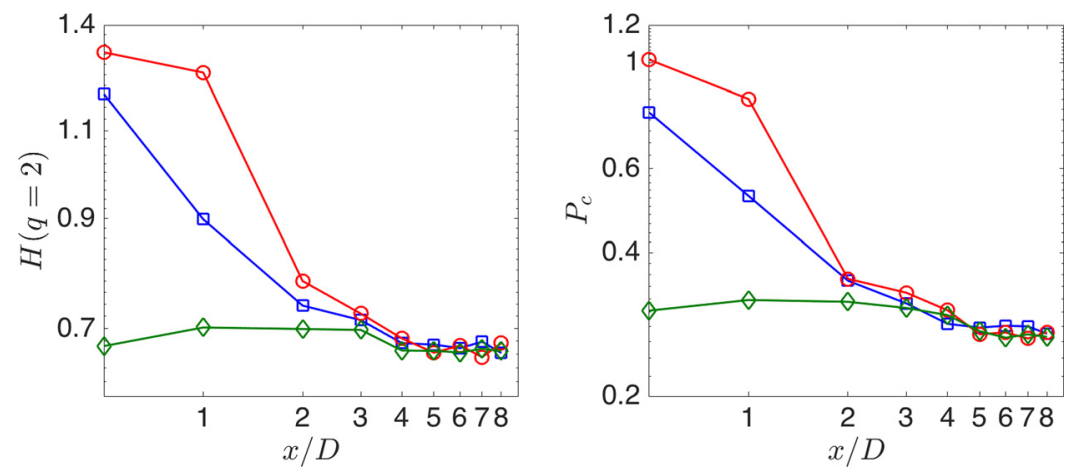

FIG. 6. The second order of the Hurst exponent, $H(q=2)$, and combination factor, $P_{c}$, at bottom tip (blue squares), hub height (red circles), and top tip (green diamonds).

$H(q=2)$ is found at $x / D=1$ and 2 at the hub height. After $4 D$ downstream, the second order of the Hurst exponent appears to stabilize although it continues to change slightly after this downstream location. At the top tip, a slight increase in $H(q=2)$ after $x / D=0.5$ is observed, and thereafter, the exponent becomes constant for the following three downstream locations. After $x / D=4, H(q=2)$ is equal to the exponent at $x / D=0.5$ and once again is approximately constant for successive downstream locations. $H(q=2)$ collapses to the same curve at the top tip, hub height, and bottom tip locations after $4 D$ from the turbine. In general, the second order of the Hurst exponent shows higher values at the near-wake region especially at hub height and bottom tip, and thereafter, decreases as the flow recovers. Thus, the stability of the $H(q=2)$ identifies when the flow has reached an equilibrium state. Following the suggestions of Morales et al. ${ }^{27}$ the results confirm the ability of the $H(q=2)$ to predict the crisis of the flow as one can see that the highest values are found at the bottom tip and hub height near the rotor, and $H(q=2)$ decreases when moving away from the rotor. Therefore, parallels between the flow as it passes through the turbine rotor and stocks during a financial crisis are suggested.

To show the acceptance of the concept of Shimizu et al. ${ }^{35}$ using the combination factor, $P_{c}$, to measure the activity of the brain, $P_{c}$ are determined to measure the activity of the dissipation. The result shows a massive decrease $(40 \%-60 \%)$ in $P_{c}$ between $0.5 \leq x / D \leq 5$ downstream of the bottom tip and hub height. Top tip regions show dissimilar behavior, where the combination factor slightly increases between $0.5 \leq x / D \leq 3$ and then decreases at the next two downstream locations. The combination factor of the top tip becomes constant after $5 D$ downstream and approximately collapses with the combination factor of the hub height and bottom tip. The maximum and minimum combination factors are found at the hub height (strong wake) and the top tip (weak wake), respectively. The turbulent kinetic energy dissipation is highly dependent on the Reynolds number; ${ }^{52}$ consequently, a strong evidence of a connection between the Reynolds number and the $P_{c}$ is drawn.

Figure 7 shows the local Reynolds numbers, $R e_{\lambda}=U \lambda / \nu$, based on Taylor-microscale, $\lambda$, the stream-wise mean velocity, $U$, and kinematic viscosity, $\nu$. The Taylor-microscale is determined using frozen field hypothesis ${ }^{52}$ as

$$
\lambda^{2}=\frac{2\left\langle u^{2}\right\rangle}{\left\langle\left(\frac{1}{U} \frac{d u}{d t}\right)^{2}\right\rangle} .
$$

The figure shows a local Reynolds number variation, where the smallest Reynolds number at $0.5 D$ downstream of the hub height and the largest at $2 D$ downstream of the top tip. The bottom tip and hub height show an increase in the Reynolds number at $0.5 \leq x / D \leq 5$ and then become approximately constant. The bottom tip, at the regions of $0.5 \leq x / D \leq 2$, shows higher $R e_{\lambda}$ than at hub height. After the two diameters downstream of the turbine, the hub height region begins to display a higher $R e_{\lambda}$ in comparison to the bottom tip region as a result of a 


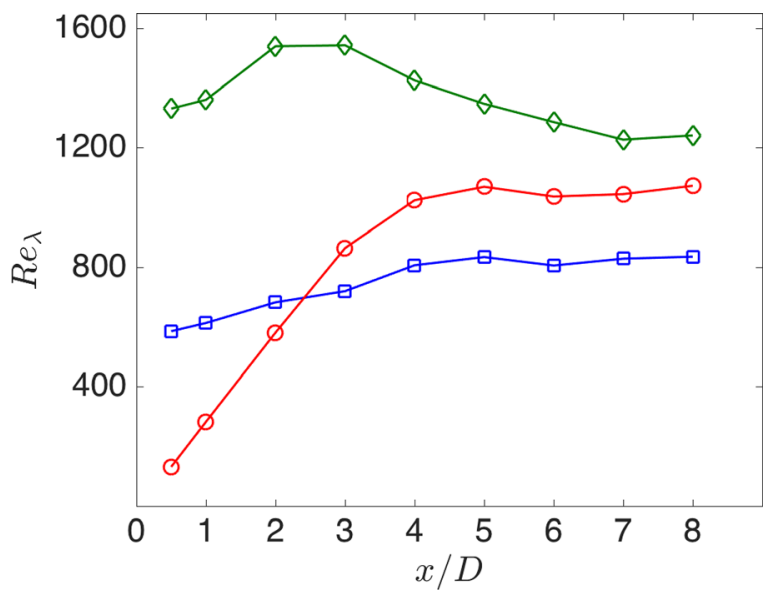

FIG. 7. Taylor microscale based Reynolds number at bottom tip (blue squares), hub height (red circles), and top tip (green diamonds).

faster recovery in the hub height region. In addition, the effects of the tower propagate downstream thus retarding the recovery in this area. The top tip region also shows an elevated $R e_{\lambda}$ at $0.5 \leq x / D \leq 2$, but thereafter decreases, specifically at $x / D \geq 3$; however, it becomes constant after $6 D$. The maximum variations between the wall-normal locations are found in the nearwake region and the variations become negligible as one moves away from the rotor. The comparison between $P_{c}$ and $R e_{\lambda}$ demonstrates that increasing the Reynolds number corresponds to a decrease in the combination factors as shown at the hub height and bottom tip. The top tip shows consistent behavior between the $P_{c}$ and $R e_{\lambda}$, where both the quantities depict a similar trend. This behavior is attributed to the rapid flow passage and creation of a shear layer at the top tip where the flow still possesses a relatively large local Reynolds number. ${ }^{1,2}$ This behavior also brings to surface questions regarding the relationship between the Reynolds number and the turbulent kinetic energy dissipation; and if the other components might be important to the total dissipations.

\section{B. Proper orthogonal decomposition analysis}

First, the normalized eigenvalue, $A_{n}$, is presented in Figure 8. For clarity, only the first 20 modes are shown. Bottom tip regions show three different decay styles, the first at the $x / D$ $=0.5$ and 1 , where the distributions show slow decay and the first 20 modes show small differences in the energy content. The second two downstream locations show a moderate decay in the distribution with the first five modes carrying a fair amount of the turbulent kinetic energy. After $4 D$, a rapid decay ensues and becomes approximately independent of the downstream locations, where the same index of the POD mode carries approximately the same amount of energy. Hub height regions also show three kinds of distributions depending on the downstream

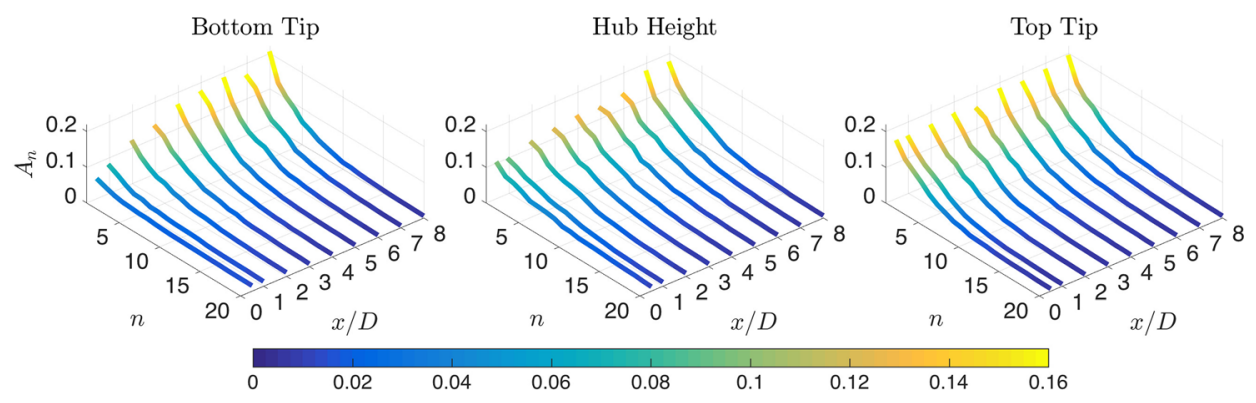

FIG. 8. Normalized turbulent kinetic energy, $A_{n}$, per modes, $n$, at the considered locations. 
location, where the first two downstream locations show a slow decay and an insignificant difference in energy content between the modes. The region between the $2 \leq x / D<6$ displays a moderate change in the energy content with increasing the index of the modes and the highest amount of energy are held in the first five modes. The last two downstream locations show the same eigenvalue distribution, where the first mode holds a remarkable amount of energy and the fast decay begins from the second mode until the tenth POD mode. After the tenth mode, there is a negligible difference in the energy content in the POD modes. The top tip exhibits approximately the same decay distributions, where the largest differences between the POD modes are found in the first ten modes and then the difference becomes travailed. The turbulent kinetic energy of the first mode increases with moving downstream, where the smallest and highest energy corresponding to the first mode are found at $x / D=0.5$ and $x / D=8$, respectively. This is due to the morphing of the flow structure from a strong wake to a quasi-recovered flow. The near-wake regions experience the effect of the turbines, where the coherent structures of the upstream flow are severed by the rotor blades, leading to the decrease in the size of these structures, in other words structures of small integral length scales. In contrast to the far-wake regions, the flow be more coherent as a result of the entrained flow from the above canopy (cf. Cal et al., ${ }^{2}$ Ali et al., ${ }^{5}$ and Melius et al. ${ }^{45}$ ). Based on wall-normal locations, the first modes at the top tip show the largest energy content compared with hub height and bottom tip especially in the near-wake region, $0.5 \leq x / D<3$. This result is due to the top tip being located near the canopy layer, where the residing coherent structures in the flow are entrained as a result of the Reynolds shear stress. The hub height displays higher energy in the first mode than bottom tip at $0.5 D$ and $1 D$ downstream of the rotor. Vortical activity in these results points towards this behavior. Aseyev and $\mathrm{Cal}^{53}$ identified the vortex content using vorticity, swirl strength, Qcriteria, $\Delta$-criteria and $\lambda_{2}$-criteria, and the results showed that the bottom tip manifests the highest activity compared with hub height and top tip in the near-wake region. The regions, $3 \leq x /$ $D<6$, also show the highest energy content in the first mode at the top tip. However, the bottom tip shows slightly higher energy in the first mode than hub height and as expected the vortices become much weaker after $3 D$. The bottom tip shows a fast recovery in comparison to the hub height.

Figure 9 tracks $50 \%$ of the cumulative energy, $B_{n}$. The largest difference between the wallnormal locations in terms of the required modes is identified at the $0.5 \mathrm{D}$ downstream the rotor, where the bottom tip requires 23 modes, in contrast to the hub height and top tip that require 9 and 5 modes, respectively. The required modes to acquire this percentage of the energy are reduced when moving downstream as a result of the entrainment mechanism that provides the rotor region with the large structures from the region above the canopy. Top tip region requires less number of modes compared to the other two locations at the $0.5 \leq x / D \leq 2$, and thereafter, the three wall-normal locations show similarity in the required modes to capture this percentage of energy. Interestingly, the top tip regions need only 4 or 5 modes as a result of the energy content in the first five modes is approximately independent on the downstream location. The amount of energy contained in individual modes tends to be larger for those in the far-wake when compared to the near-wake. Furthermore, the extent of scales contained in the near versus far-wake is rather different as the integral scale is approximately 5 times larger in the far-wake at $x / D=5$.

Figure 10 displays $75 \%$ of normalized successive energy, where the bottom tip necessitates more modes than the hub height and the top tip between $0.5 \leq x / D \leq 4$, whereas the top tip demands the least out of the considered vertical locations. After $4 D$, the required modes become independent on the wall-normal and streamwise locations. Top tip regions show approximate similarity at $x / D>1$ where the number of modes is approximately unchanged. Downstream the hub height, the required modes show a slow change and become approximately constant after $4 D$. The rapid variations between the downstream locations are found at the bottom tip especially at $0.5 \leq x / D \leq 4$. After $5 D$ downstream of the bottom tip, the variation between the locations is negligible.

To capture $95 \%$ of energy, the bottom tip demands more modes compared to other two locations between $0.5 \leq x / D \leq 5$, with successive downstream locations demanding less modes 


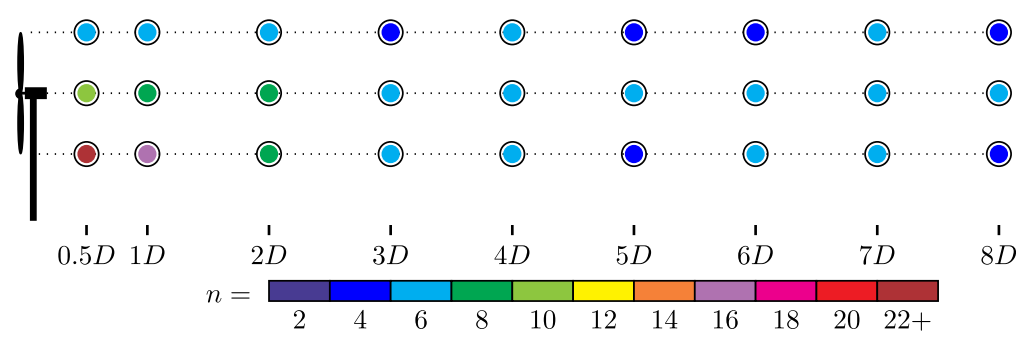

FIG. 9. Corresponding modes for $50 \%$ reconstruction of the turbulent kinetic energy.

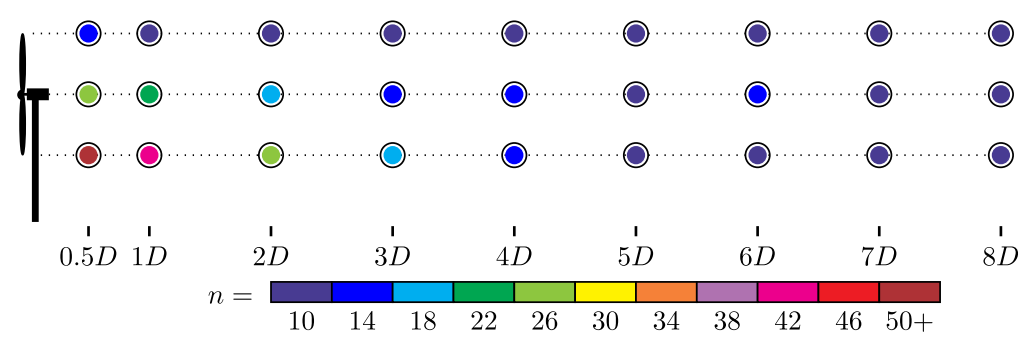

FIG. 10. Corresponding modes for $75 \%$ reconstruction of the turbulent kinetic energy.

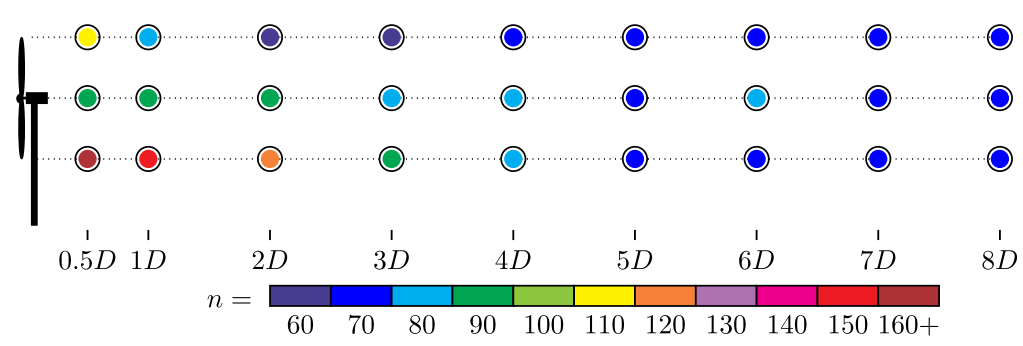

FIG. 11. Corresponding modes for $95 \%$ reconstruction of the turbulent kinetic energy.

than the hub height. The top tip also shows less required modes than the bottom tip and hub height with the exception of $x / D=0.5$, as shown in Figure 11. Even though for $50 \%$ and $75 \%$, the top tip required less modes than the hub height and bottom tip; at 95\%, it requires the most modes, surpassing the other locations. This points towards the importance of the small scales and/or energy content at high mode numbers. Two different regimes are identified at the top tip, the first is noticed at the region of $0.5 \leq x / D \leq 3$, where the required mode decreases with increasing $x / D$ and shows a significant variation in the number of the modes. The second regime begins after $3 D$, where the amount of required modes increases with increasing $x / D$ and the rate at which it increases continues in the limit of 66-68 modes. This behavior is attributed to the embedded tip vortices in this area. The oscillation that is observed in Figure 6 after $x / D>5$ is due to the exchange in energy of the small scales. Through the different percentages of the successive energy, it is of interest to show that the bottom tip shows rapid change dependent on the mode requirement compared with the hub height that shows small or slow variation in downstream locations.

\section{Multifractal analysis of the reconstructed flow field}

In combining the multifractal framework of the turbulent kinetic energy dissipation with proper orthogonal decomposition of the stochastic signals, a reconstruction of the velocity is sought out to show the dissipation. Reduced order techniques depend on the energy cascade, where the energy flows from the low index POD eigenfunctions to the higher index.$^{54}$ Dividing the velocity into large and small scales facilitates the ability to understand how the scales 

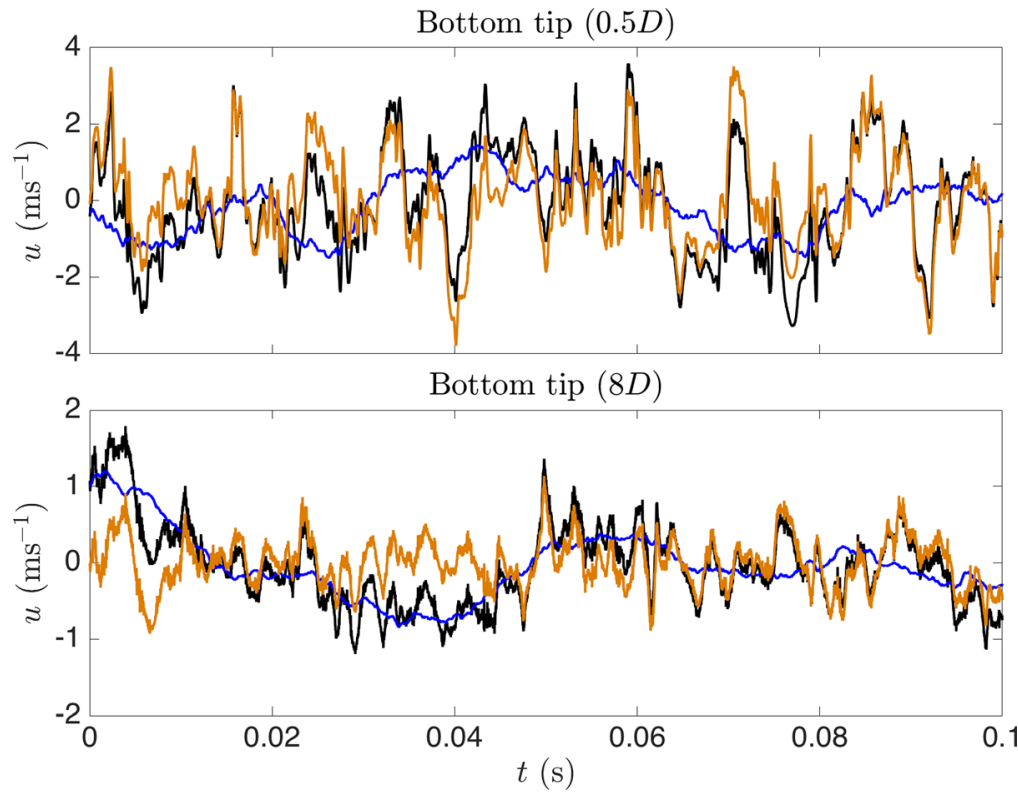

FIG. 12. Streamwise fluctuation velocity at $0.5 D$ and $8 D$ downstream of the bottom tip. Original signal (-), the reconstructed velocity from the first 20 POD mode (blue lines), and next 460 modes (orange lines).

dissipate and differ from the original signal in terms of the multifractal characteristics. The stochastic velocity can be reconstructed using the specific number of the eigenfunctions and the projection of the velocity on the POD modes. Here, streamwise velocities are rebuilt from the large and small scales, considering the first 20 modes (referenced as large scale) carry a large percentage of the energy as previously explained in Figure 8 and the rest (referenced as small scale) contain the small scales in the finite domain. The reconstructed streamwise velocities are fed into dissipation equation in order to determine the turbulence kinetic energy dissipation, thereafter applying the multifractal algorithm. For the rest of the analysis, it will be considered that the large and small scale velocities that are fed into dissipation equation are referenced as the large scale dissipation and small scale dissipation, respectively.

Figure 12 compares the original and reconstructed velocities at $0.5 D$ and $8 D$ downstream of the bottom tip. For clarity, the figure presents only $0.1 \mathrm{~s}$ of the $100 \mathrm{~s}$ total length of the timeseries data. The reconstructed velocity from the large scale modes passes over the original signal without taking the shape of the fluctuation. By contrast, the small scales exactly converge to the shape of the original velocity.

The multifractal spectra of the dissipation of the reconstructed signal from the first 20 POD modes are presented in Figure 13. Although the energy level of the first 20 modes is dependent on the downstream and wall-normal locations, the singularity spectrum exhibits approximately similar distribution throughout the downstream locations and the multifractality is moved to a

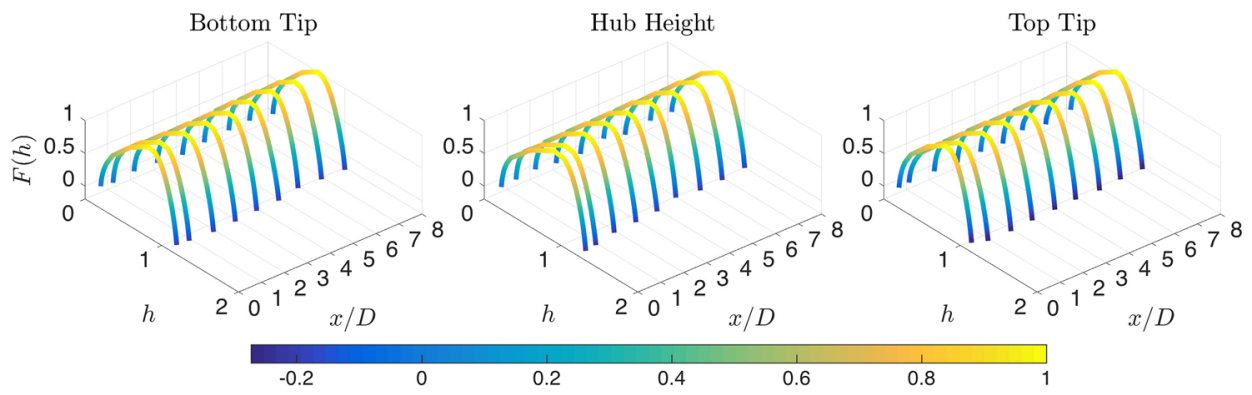

FIG. 13. Distribution of the singularity spectrum of the large scale at the considered locations. 


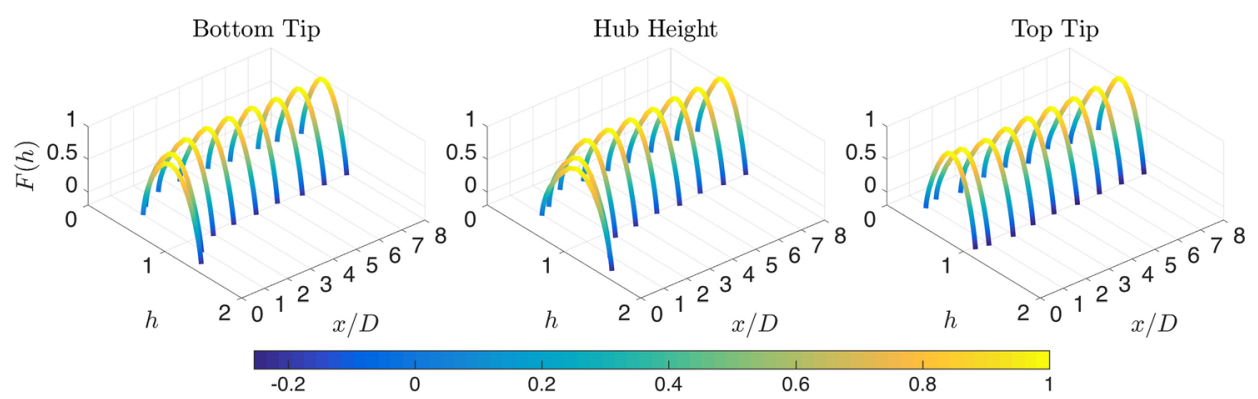

FIG. 14. Distribution of the singularity spectrum of the small scale at the considered locations.

null Hölder exponent. As explained in Sec. IV A, when the minimum Hölder exponent is reduced, the complexity of the signal is reduced as well, thus the reconstructed signal is less complex than the original signal. This is a consequence of reducing the interaction between the small and large scales.

In Figure 14, the singular spectra of the small scales are identified, where they display approximately the same trend that is observed on the spectra of the original signal, see Figure 4. The bottom tip displays a multifractal spectrum showing higher Hölder exponents at $x / D=0.5$ and 1 , and subsequently, the distributions contain relatively lower Hölder exponents. The spectra, after $3 D$ downstream of the bottom tip, become approximately independent of the spatial locations. A similar behavior is observed at the hub height region, where the highest Hölder exponent is reached at the spectrum of $0.5 D$. At the top tip, all spectra distributions are located at the same limits of the Hölder exponent.

To examine the difference between the original signal and the reconstructed signals, the multifractal spectra are overlaid as illustrated in Figure 15. Three different downstream locations are chosen to verify the consistency or inconsistency between signals located at $x / D=0.5$, 3 , and 8 . The major differences are noticed between the signals at the $0.5 D$ downstream of the bottom tip and hub height, where the original signal moves toward the highest Hölder exponent direction and the large scale signal moves toward the minimum Hölder exponent. The singularity spectrum constructed via small scales lies between them. A slight variation between the
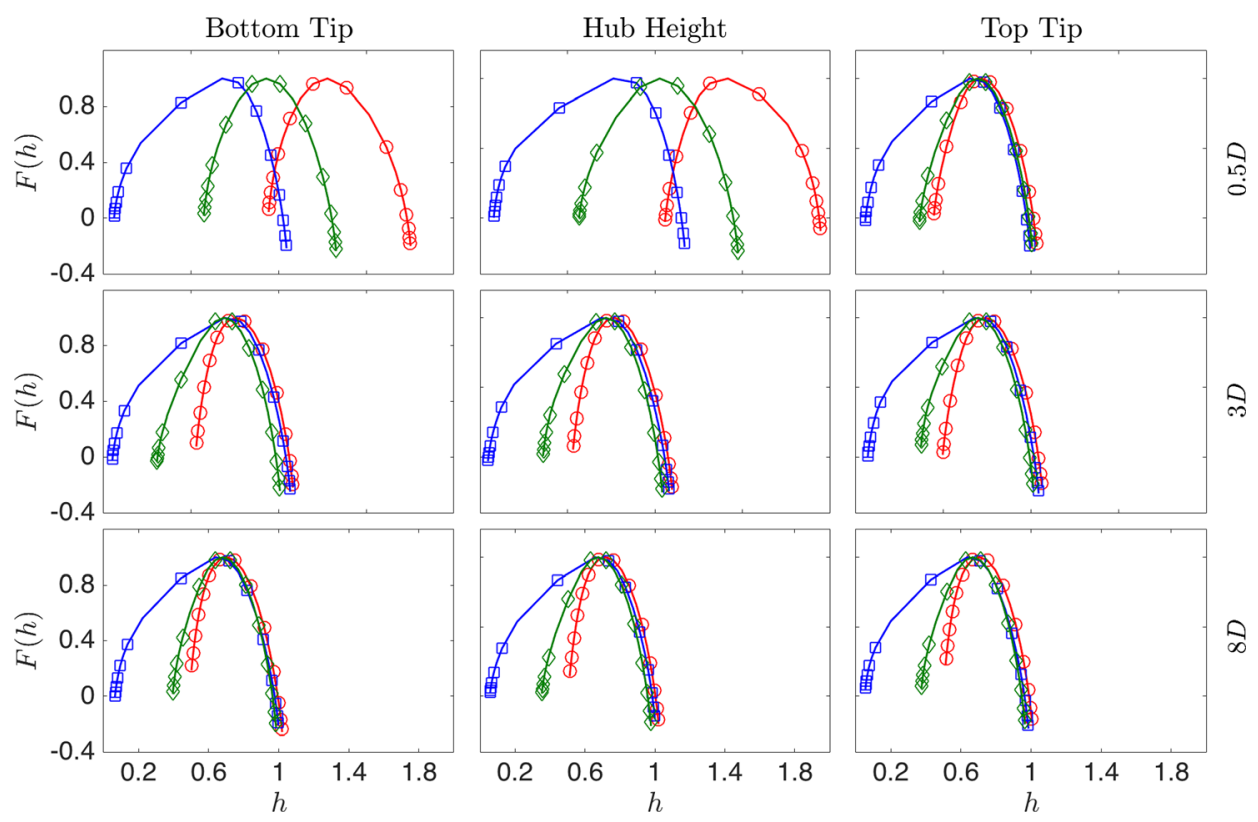

FIG. 15. Singularity spectrum of the bottom tip, hub height, and top tip at $0.5 D, 5 D$, and $8 D$. Original (red circles), large scale (blue squares), and small scale (green diamonds) signals. 
small scale and the original signals is observed at the top tip. Thus, the variations between the small scale and original signals decrease with increasing the downstream locations. Interestingly, the right tail of the large scale and small scale spectra are approximately coincident with the original distribution, especially at three and eight diameters downstream the turbine. The right tails of the three signals are coincident at the top tip and contain small variation at the bottom tip and hub height. For the left tails, the large scale signal deviates far from the original signal while the small scale signal carries moderate variations.

Figure 16 outlines the Hurst exponents determined from the original, large scale and small scale dissipations. The large scale dissipations show more multifractal structures than the other two signals, where the largest difference between the maximum and minimum orders, $q= \pm 15$, is found in the large scale signals at hub height, bottom tip, and top tip locations. Half diameter downstream of the bottom tip and hub height, the largest deviations in the Hurst exponents' distribution occur, where the original dissipation is shifted to the highest exponent and the large scale dissipation is towards the smallest exponent. Thus, the small scale dissipation is located between them. The Hurst exponent distribution of the small scale dissipation becomes closer to the original signal moving further downstream. The top tip regions show the same multifractal structure and the Hurst exponents of the three signals are approximately independent of the location downstream the rotor. Furthermore, the three dissipation signals, with an exception $0.5 \mathrm{D}$ downstream of the hub height and bottom tip, are coincident for all negative moments and the difference is only in the positive orders. In general, the multifractal structures increase with the reconstructed signals and this result is consistent with the result found in Ref. 43, where the POD increases the multifractality of the velocity signal.

Figure 17 presents the second order of the Hurst exponents and the combination factor of the large scale and small scale dissipation. The second order of the Hurst exponents of the large scale is approximately constant where the deviation of order, $\mathcal{O}\left(10^{-2}\right)$, is observed. Therefore, the second order of the Hurst exponents is independent of the downstream and the wall-normal locations. The small scale dissipations follow the same trend that is noticed in the dissipation of the original signal as shown in Figure 6. The bottom tip and hub height exhibit decrease in the $H(q=2)$ with increasing the downstream location, and reach an approximately constant value after $x / D=3$. Top tip shows a slight increase in the $H(q=2)$ at $1 D$ and $2 D$ and then
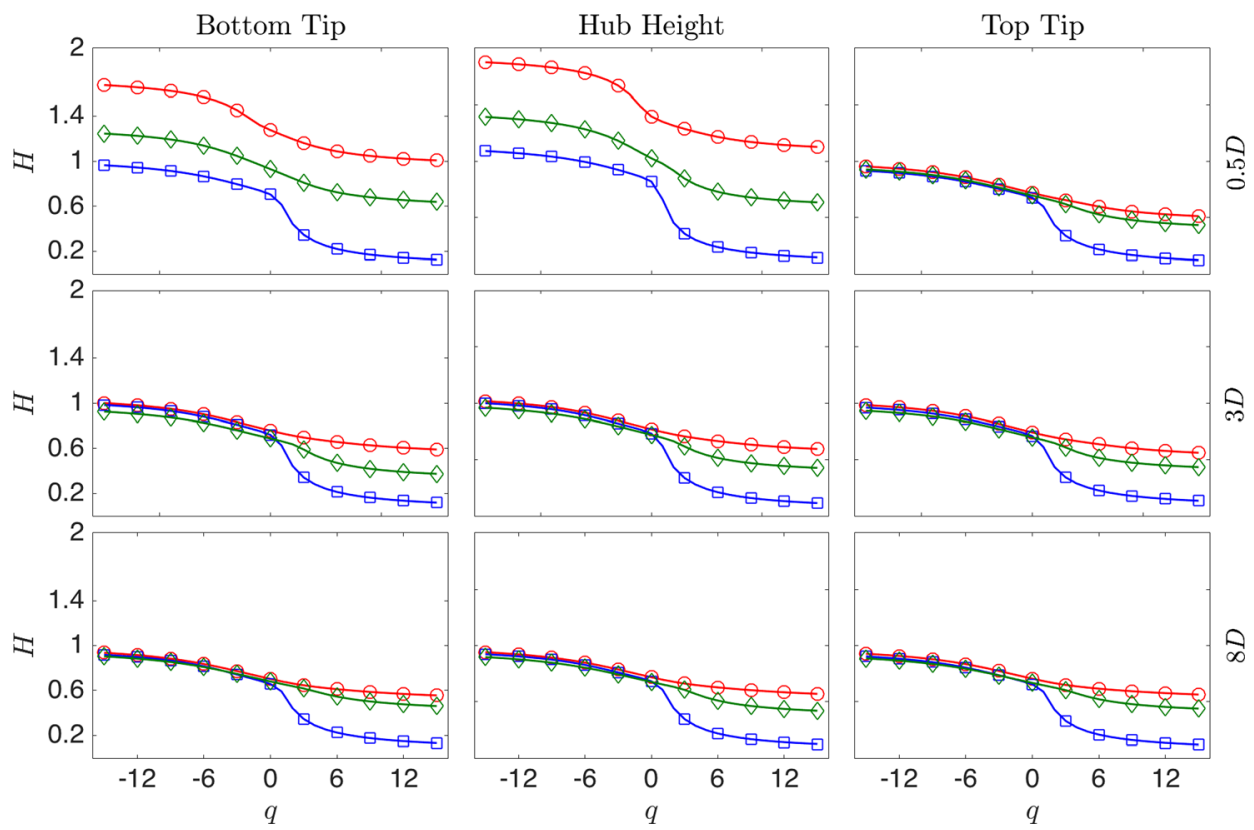

FIG. 16. Hurst exponents of the bottom tip, hub height, and top tip at $0.5 D, 5 D$, and $8 D$. Original (red circles), large scale (blue squares), and small scale (green diamonds) signals. 

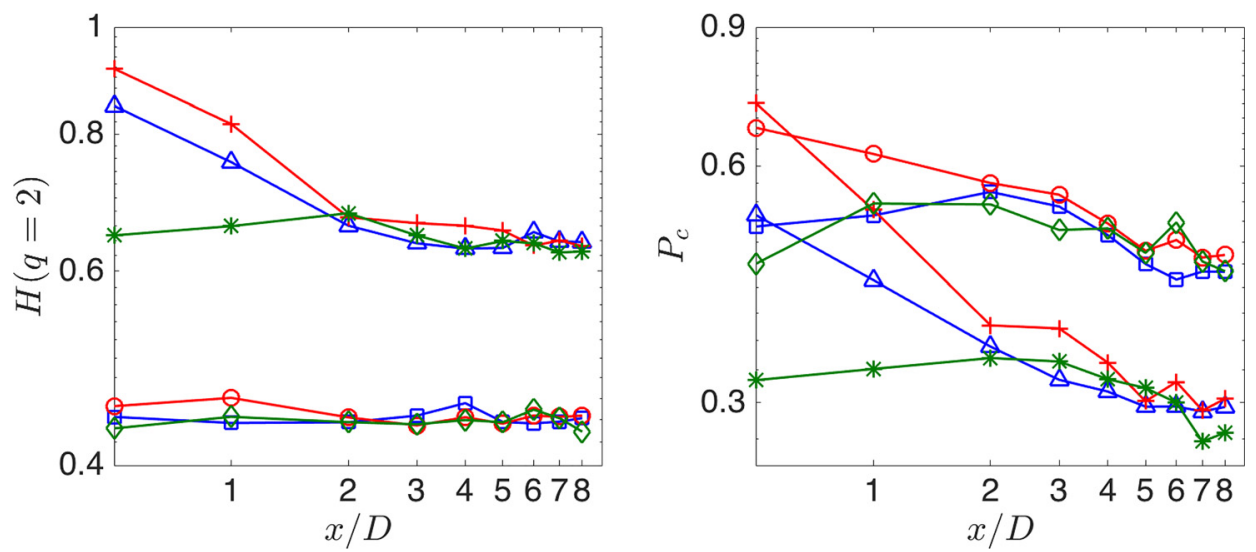

FIG. 17. The second order of the Hurst exponent, $H(q=2)$, and combination factor, $P_{c}$, at: bottom tip-large scale ((blue squares), bottom tip-small scale (blue triangles), hub height-large scale (red circles), hub height-small scale (red crosses), top tip-large scale (green diamonds), and top tip-small scale (green stars).

decreases while oscillating around the constant value of 0.62 . Downstream locations show the largest combination factor, $P_{c}$, of the large and small scale dissipations at the hub height. The bottom and top tips alternate having the largest $P_{c}$ while moving downstream, for example, at the region of $0.5 \leq x / D \leq 2$, the small scale dissipation at the bottom tip exceeds the dissipation at the top tip, whereas at $3 \leq x / D \leq 5$ the trend is reversed. The dissipation at the bottom tip displays different trends in large and small scale signals, where the large scale exhibits increasing of the $P_{c}$ at the region $0.5 \leq x / D \leq 3$ and then begins decreasing with increasing downstream distance. The region between $6 \leq x / D \leq 8$ displays approximately the same combination factors. By contrast, the small scale dissipation signal exhibits decreasing in the $P_{c}$ at the region $0.5 \leq x / D \leq 3$ and then it becomes constant. Downstream of the hub height shows that the largeand small scale dissipations coincide in conduct, where both dissipations decrease while moving downstream from the rotor and then become approximately constant after 5 diameters downstream. However, both dissipation signals show a minute increase in the $P_{c}$ at a 6 diameter downstream. The large scale dissipation signal shows increasing $P_{c}$ at 1 and 2 diameters downstream of the top tip, and oscillates around the $P_{c} \approx 0.52$. Similarly, the small scale dissipations also show increasing $P_{c}$ between the $1 D$ and $3 D$ downstream and then decreasing until the $8 D$ location, where there exists a slight increase in the $P_{c}$. Furthermore, between 6 and 7 diameters downstream of the top tip, both large- and small scales show a noticeable change in the combination factor compared with the other neighbor locations.

\section{CONCLUSION}

Hot-wire anemometry data gathered in a wind tunnel experiment were used to study the multifractal characteristics of the turbulent kinetic energy dissipation behind the center turbine in the exit row of a wind turbine array. Focused-based multifractal wavelet leader is used to quantify the singularity spectrum, Hurst exponents, and the combination factor as dependent on the spatial locations. The strength of the multifractal system is determined through the singularity spectrum and Hurst exponents. The hub height and bottom tip contain high multifractality, especially $0.5 D$ and $1 D$ downstream of the rotor. The multifractality decreases as the downstream distance is increased. Top tip regions display a unified behavior of the multifractal structure, where the spectra are located approximately at the same limit of the Hölder exponent. Although the three wall-normal locations reveal the multifractality at the nine downstream positions, the top tip exhibits a reduced multifractality compared to the hub height and bottom tip, especially in the near-wake region. Thereafter, the three locations approximately converge in their multifractal distributions. The Hurst exponents also exhibit the same behavior shown in the singularity spectrum, where the maximum exponents are found at $0.5 D$ and $1 D$ downstream 
of the hub height and bottom tip. The top tip region contains small variations in the order based Hurst exponent. The second order of the Hurst exponent displays higher values at the hub height and the bottom tip, especially at near-wake regions. In accordance with another behavior, after $5 D$, the same exponents are yielded. The $H(q=2)$ is used as an indicator about the flow state and its development, which in this case pertains to a wake or a kind of recovered flow. The combination factor, $P_{c}$, also exhibits the same trend that is observed in the $H(q=2)$. Based on the multifractal parameters, the far-wake regions are characterized by approximately constant singular spectrum and low multifractality or intermittency. In contrast, the near-wake region that is characterized with high multifractality are induced by the rotor and propagated downstream the wind turbines. The degree of multifractality correlates with the degree of perturbation in the flow. Therefore, multifractal parameters can efficiently distinguish between the near- and far-wake regions and reveal the wake propagation. In addition, the power production that is highly affected by the flow state can be in correlation with the combination factor, where both can predict the activity of the flow field.

Snapshot proper orthogonal decomposition is used to detect the coherent and incoherent structures in the flow field. The turbulent kinetic energy is presented as normalized energy per mode, $A_{n}$, and a cumulative energy, $B_{n}$. The first mode at the top tip carries higher turbulent kinetic energy than the bottom tip and hub height. Thus, the amount of energy per mode is approximately independent at the locations downstream of the top tip. The minimum amount of the energy in the first mode is found at the $0.5 D$ and $1 D$ downstream of the hub height and bottom tip which also show the slow drainage energy. Comparing the three wall-normal locations in the near-wake regions, the top tip shows a fast convergence profile of the successive energy. In contrast, the far-wake regions show approximately the same convergence profile.

Stochastic velocities are reconstructed using the POD eigenfunctions and then applying the multifractal analysis to measure the impact of the changing of the flow structures on the multifractality. The singularity spectrum of the large scale dissipation shows the same distributions and is independent of physical location. In contrast, the multifractal spectrum of the small scale dissipation shows approximately the same behavior of the original signal. The three original signals are overlaid with the large and small scale signals to test the multifractality. The result shows that the three signals coincide at the right tail of the spectra and the deviation happens only at the left tail. However, the three signals show maximum differences at $0.5 D$ downstream of the bottom tip and hub height. Thus, the deviation between the small scale and original signal dissipation decreases with the moving downstream of the rotor. The Hurst exponents confirm the result that is noticed in the singularity spectrum. The $H(q=2)$ is approximately constant for the dissipation of the large scale at the hub height, bottom and top tips. In contrast, the small scale dissipations decrease in the $H(q=2)$ for $x / D<3$ downstream of the hub height and bottom tip, and become constant thereafter. The main goal of the current study was to determine the multifractality of the turbulent kinetic energy dissipation in the wind farm. The second order Hurst exponent and the combination factor as shown in this study have the ability to demonstrate the changes between the near- and far-wake regions, predict the developed and developing flow, and show the activity of the energy dissipation. Therefore, these can be useful to determine the maximum energy producing spacing between the wind turbines and used as design criteria for wind farm sitting besides the produced power and economic constraints. Further studies can investigate the effects of the thermal stratification on the multifractal characteristics and flow structures.

\section{ACKNOWLEDGMENTS}

The authors are grateful to the National Science Foundation (Grant No. CBET-1034581) for funding part of this research.

${ }^{1}$ L. P. Chamorro and F. Porté-Agel, "Turbulent flow inside and above a wind farm: a wind-tunnel study," Energies 4, 1916-1936 (2011).

${ }^{2}$ R. B. Cal, J. Lebrón, L. Castillo, H.-S. Kang, and C. Meneveau, "Experimental study of the horizontally averaged flow structure in a model wind-turbine array boundary layer," J. Renewable Sustainable Energy 2, 013106 (2010). 
${ }^{3}$ M. Calaf, C. Meneveau, and J. Meyers, "Large eddy simulation study of fully developed wind-turbine array boundary layers," Phys. Fluids 22(1), 015110 (2010).

${ }^{4} \mathrm{~J}$. Meyers and C. Meneveau, "Optimal turbine spacing in fully developed wind-farm boundary layers," Wind Energy 15, 305-317 (2012).

${ }^{5}$ N. Ali, A. Aseyev, M. Melius, M. Tutkun, and R. B. Cal, Evaluation of Higher Order Moments and Isotropy in the Wake of a Wind Turbine Array (Springer, 2017), pp. 273-292.

${ }^{6} \mathrm{~B}$. B. Mandelbrot, The Fractal Geometry of Nature (Macmillan, 1983).

${ }^{7}$ J. C. Sprott, Chaos and Time-Series Analysis (Oxford University Press, 2003), p. 69.

${ }^{8}$ Y. Shimizu, M. Barth, C. Windischberger, E. Moser, and S. Thurner, "Scaling structure of the surface layer of diffusionlimited aggregates," Phys. Rev. Lett. 56(8), 854 (1986).

${ }^{9}$ E. A. Ihlen, "Introduction to multifractal detrended fluctuation analysis in Matlab," Fractal Anal. 3, 97 (2012).

${ }^{10}$ A. Eke, P. Herman, C. Windischberger, J. Bassingthwaighte, G. Raymond, D. Percival, M. Cannon, I. Balla, and C. Ikrényi, "Physiological time series: Distinguishing fractal noises from motions," Pflügers Arch. 439(4), 403-415 (2000).

${ }^{11}$ S. Jaffard, B. Lashermes, and P. Abry, "Wavelet leaders in multifractal analysis," in Wavelet Analysis and Applications (Springer, 2006), pp. 201-246.

${ }^{12}$ F. Toschi, G. Amati, S. Succi, R. Benzi, and R. Piva, "Intermittency and structure functions in channel flow turbulence," Phys. Rev. Lett. 82(25), 5044 (1999).

${ }^{13}$ E. Falcon, S. Fauve, and C. Laroche, "Observation of intermittency in wave turbulence," Phys. Rev. Lett. 98(15), 154501 (2007).

${ }^{14}$ S. Futatani, S. Bankadda, Y. Nakamura, and K. Kondo, "Multiscaling dynamics of impurity transport in drift-wave turbulence," Phys. Rev. Lett. 100(2), 025005 (2008).

${ }^{15}$ U. Frisch and G. Parisi, "Turbulence and predictability of geophysical fluid dynamics and climate dynamics," in Proceedings of the International School of Physics 'Enrico Fermi,' Course LXXXVIII, Varenna, edited by M. Ghil (North-Holland, New York, 1985).

${ }^{16} \mathrm{~B}$. B. Mandelbrot, "Intermittent turbulence in self-similar cascades: Divergence of high moments and dimension of the carrier," J. Fluid Mech. 62(2), 331-358 (1974).

${ }^{17}$ C. Meneveau and K. R. Sreenivasan, "Simple multifractal cascade model for fully developed turbulence," Phys. Rev. Lett. 59(13), 1424 (1987).

${ }^{18}$ L. P. Chamorro, J. Hong, and C. Gangodagamage, "On the scale-to-scale coupling between a full-scale wind turbine and turbulence," J. Turbul. 16, 617-632 (2015).

${ }^{19}$ N. Ali, A. S. Aseyev, and R. B. Cal, "Structure functions, scaling exponents and intermittency in the wake of a wind turbine array," J. Renewable Sustainable Energy 8(1), 013304 (2016).

${ }^{20}$ C.-K. Lee, L.-C. Juang, C.-C. Wang, Y.-Y. Liao, C.-C. Yu, and Y.-C. Liu, "Scaling characteristics in ozone concentration time series (OCTS)," Chemosphere 62(6), 934-946 (2006).

${ }^{21}$ Y. Tessier, S. Lovejoy, P. Hubert, D. Schertzer, and S. Pecknold, "Multifractal analysis and modeling of rainfall and river flows and scaling, causal transfer functions," J. Geophys. Res.: Atmos. 101(D21), 26427-26440, doi:10.1029/96JD01799 (1996).

${ }^{22}$ P. Shang, Y. Lu, P. Hubert, and S. Kamae, "Detecting long-range correlations of traffic time series with multifractal detrended fluctuation analysis," Chaos, Solitons Fractals 36(1), 82-90 (2008).

${ }^{23}$ X. Sun, H. Chen, Y. Yuan, and Z. Wu, "Predictability of multifractal analysis of Hang Seng stock index in Hong Kong," Physica A 301(1), 473-482 (2001).

${ }^{24} \mathrm{H}$. Arevalo, B. Rodriguez, and N. Trayanova, "Arrhythmogenesis in the heart: Multiscale modeling of the effects of defibrillation shocks and the role of electrophysiological heterogeneity," Chaos 17(1), 015103 (2007).

${ }^{25}$ Y. Wei and D. Huang, "Multifractal analysis of SSEC in Chinese stock market: A different empirical result from Heng Seng index," Physica A 355(2), 497-508 (2005).

${ }^{26}$ L. Zunino, A. Figliola, B. M. Tabak, D. G. Pérez, M. Garavaglia, and O. A. Rosso, "Multifractal structure in LatinAmerican market indices," Chaos, Solitons Fractals 41(5), 2331-2340 (2009).

${ }^{27}$ R. Morales, T. Di Matteo, R. Gramatica, and T. Aste, "Dynamical generalized Hurst exponent as a tool to monitor unstable periods in financial time series," Physica A 391(11), 3180-3189 (2012).

${ }^{28}$ J. W. Kantelhardt, "Fractal and multifractal time series," in Mathematics of Complexity and Dynamical Systems (Springer, 2012), pp. 463-487.

${ }^{29}$ L. E. Calvet and A. J. Fisher, “A Multifractal Model of Assets Returns," NYU Working Paper No. FIN-99-072, 1999.

${ }^{30} \mathrm{P}$. Mukli, Z. Nagy, and A. Eke, "Multifractal formalism by enforcing the universal behavior of scaling functions," Physica A 417, 150-167 (2015).

${ }^{31}$ T. Di Matteo, T. Aste, and M. M. Dacorogna, "Long-term memories of developed and emerging markets: Using the scaling analysis to characterize their stage of development," J. Banking Finance 29(4), 827-851 (2005).

${ }^{32}$ R. V. Solé and S. C. Manrubia, "Self-similarity in rain forests: Evidence for a critical state," Phys. Rev. E 51(6), 6250 (1995).

${ }^{33}$ L. A. Amaral, P. C. Ivanov, N. Aoyagi, I. Hidaka, S. Tomono, A. L. Goldberger, H. E. Stanley, and Y. Yamamoto, "Behavioral-independent features of complex heartbeat dynamics," Phys. Rev. Lett. 86(26), 6026 (2001).

${ }^{34} \mathrm{C}$. Meneveau and K. R. Sreenivasan, "Measurement of $\mathrm{f}(\alpha)$ from scaling of histograms, and applications to dynamical systems and fully developed turbulence," Phys. Lett. A 137(3), 103-112 (1989).

${ }^{35}$ Y. Shimizu, M. Barth, C. Windischberger, E. Moser, and S. Thurner, "Wavelet-based multifractal analysis of fMRI time series," Neuroimage 22(3), 1195-1202 (2004).

${ }^{36}$ J. L. Lumley, "The structure of inhomogeneous turbulent flows," in Atmospheric Turbulence Radio Wave Propagation (Nauka, Moscow, 1967).

${ }^{37}$ L. Sirovich, "Turbulence and the dynamics of coherent structures. Part I: Coherent structures," Q. Appl. Math. 45(3), 561-571 (1987).

${ }^{38}$ N. Hamilton, M. Tutkun, and R. B. Cal, "Wind turbine boundary layer arrays for Cartesian and staggered configurations: Part II, low-dimensional representations via the proper orthogonal decomposition," Wind Energy 18(2), 297-315 (2015). 
${ }^{39} \mathrm{C}$. VerHulst and C. Meneveau, "Large eddy simulation study of the kinetic energy entrainment by energetic turbulent flow structures in large wind farms," Phys. Fluids 26(2), 025113 (2014).

${ }^{40} \mathrm{~N}$. Hamilton, M. Tutkun, and R. B. Cal, "Low-order representations of the canonical wind turbine array boundary layer via double proper orthogonal decomposition," Phys. Fluids 28(2), 025103 (2016).

${ }^{41}$ P. Moin and R. D. Moser, "Characteristic-eddy decomposition of turbulence in a channel," J. Fluid Mech. 200, 471-509 (1989).

${ }^{42}$ Y. Wu and K. T. Christensen, "Spatial structure of a turbulent boundary layer with irregular surface roughness," J. Fluid Mech. 655, 380-418 (2010).

${ }^{43}$ L. Ukeiley, M. Varghese, M. Glauser, and D. Valentine, "Multifractal analysis of a lobed mixer flowfield utilizing the proper orthogonal decomposition," AIAA J. 30(5), 1260-1267 (1992).

${ }^{44} \mathrm{G}$. V. Iungo and E. Lombardi, "A procedure based on proper orthogonal decomposition for time-frequency analysis of time series," Exp. Fluids 51(4), 969-985 (2011).

${ }^{45}$ M. Melius, M. Tutkun, and R. B. Cal, "Identification of Markov process within a wind turbine array boundary layer," J. Renewable Sustainable Energy 6(2), 023121 (2014).

${ }^{46}$ C. Meneveau and K. R. Sreenivasan, "The multifractal spectrum of the dissipation field in turbulent flows," Nucl. Phys. B-Proc. Suppl. 2, 49-76 (1987).

${ }^{47}$ L. P. Chamorro, M. Guala, R. E. A. Arndt, and F. Sotiropoulos, "Turbulent flow inside and above a wind farm: A windtunnel study," J. Turbul. 13, N27 (2012).

${ }^{48} \mathrm{E}$. Li, X. Mu, G. Zhao, and P. Gao, "Multifractal detrended fluctuation analysis of streamflow in the Yellow River Basin, China," Water 7(4), 1670-1686 (2015).

${ }^{49}$ Y. Seo and S. Lyu, "Multifractal characteristics of the jet turbulent intensity depending on the outfall nozzle geometry," Stochastic Environ. Res. Risk Assess. 30, 653-664 (2016).

${ }^{50}$ W. M. Macek, "Chaos and multifractals in the solar wind," Adv. Space Res. 46(4), 526-531 (2010).

${ }^{51}$ A. N. Kolmogorov, "Dissipation of energy in locally isotropic turbulence," Proc. Math. Phys. Sci. 32(1), 15-17 (1941).

${ }^{52}$ S. B. Pope, Turbulent Flows (Cambridge University Press, 2001).

${ }^{53}$ A. S. Aseyev and R. B. Cal, "Vortex identification in the wake of a model wind turbine array," J. Turbul. 17, 357-378 (2015).

${ }^{54}$ J. Borggaard, A. Duggleby, T. Iliescu, and Z. Wang, "Reduced-order modeling of turbulent flows," in Proceedings of the MTNS (2008). 